\title{
A novel FBN1 mutation causes autosomal dominant Marfan syndrome
}

\author{
YING XIAO $^{1 *}$, XIAOQI LIU ${ }^{2 *}$, XIAOXIN GUO ${ }^{2 *}$, LIPING LIU $^{1}$, LINXIN JIANG $^{2}$, QI WANG ${ }^{1}$ and BO GONG ${ }^{2}$ \\ ${ }^{1}$ Department of Ophthalmology, Shandong Provincial Hospital Affiliated to Shandong University, Jinan, Shandong 250021; \\ ${ }^{2}$ Sichuan Provincial Key Laboratory for Disease Gene Study, Hospital of University of Electronic Science \\ and Technology of China and Sichuan Provincial People's Hospital, School of Medicine, University \\ of Electronic Science and Technology of China, Chengdu, Sichuan 610072, P.R. China
}

Received December 28, 2016; Accepted August 4, 2017

DOI: $10.3892 / \mathrm{mmr} .2017 .7544$

\begin{abstract}
Marfan syndrome (MFS) is an inherited and systemic disorder. It has been reported that mutations in the fibrillin-1 gene (FBN1) account for $\sim 90 \%$ of autosomal dominant cases of MFS. This study was conducted to screen mutations of FBN1 in a Chinese family with autosomal dominant MFS; four individuals including two patients with MFS were recruited. The family members underwent complete physical, cardiovascular and ophthalmologic examinations. Genomic DNA samples were collected from the family along with 383 unrelated healthy subjects. FBN1 coding regions were amplified by polymerase chain reaction and analyzed by direct sequencing. SIFT and PolyPhen- 2 were used to predict the possible structural and functional alterations of the protein. A novel heterozygous mutation c.1708 $\mathrm{T}>\mathrm{G}$ (p.C570G) in exon 14 was identified, which led to a substitution of cysteine by glycine at codon 570 (p.C570G). The mutation was identified as being associated with the MFS phenotype in the affected members of this family. However, the unaffected family members and the 383 normal controls lacked the mutation. Multiple sequence alignment of the human FBN1 protein revealed that this novel mutation occurred within a
\end{abstract}

Correspondence to: Dr Bo Gong, Sichuan Provincial Key Laboratory for Disease Gene Study, Hospital of University of Electronic Science and Technology of China and Sichuan Provincial People's Hospital, School of Medicine, University of Electronic Science and Technology of China, 32 Road West 2, the First Ring, Chengdu, Sichuan 610072, P.R. China

E-mail: gongbo2007@hotmail.com

Dr Qi Wang, Department of Ophthalmology, Shandong Provincial Hospital Affiliated to Shandong University, 324 Jingwu Road, Jinan, Shandong 250021, P.R. China

E-mail: drwangqi@hotmail.com

*Contributed equally

Key words: Marfan syndrome, fibrillin-1, autosomal dominant, heterozygous mutation highly conserved region of the FBN1 protein across different species and may induce structural alterations in this functional domain. The spectrum of MFS-associated mutations in the FBN1 gene has been enriched from this study; this may improve understanding of the molecular pathogenesis and clinical diagnosis of MFS.

\section{Introduction}

Marfan syndrome (MFS) is an autosomal dominant hereditary disease comprising a disorder of fibrous connective tissue involving the ocular, skeletal and cardiovascular systems (1). According to the Ghent criteria, patients with malfunctions of at least two organ systems could be diagnosed with MFS (2). Aortic root dilatation/dissection and lens dislocation were two cardinal manifestations to establish an unequivocal diagnosis of MFS in patients with positive family history. Due to the large clinical variability of MFS, and several other connective tissue disorders with comparable clinical features, distinguishing MFS from those similar syndromes is still challenging.

Increasing evidence indicates that heredity holds a key role in the development of MFS. It has been reported that MFS generally results from mutations in the human fibrillin-1 $(F B N 1)$ gene $(3,4)$. At present, $>3,000$ mutations have been identified in relation to MFS. Most mutations are specific to a family with MFS, whereas $\sim 10 \%$ of FBN1 mutations are shared by different families (5). Located at chromosome $15 \mathrm{q}-21.1$ with 65 exons, the $F B N 1$ gene encodes a secreted $350 \mathrm{kDa}$ glycoprotein (6). Human FBN1 protein shares conserved sequences with other species. FBN1 protein constitutes extracellular microfibrils and controls the stability, as well as the microfibril assembly. Mutations within the FBN1 gene may disrupt microfibril formation, leading to abnormalities of fibrillin and eventually weakening the connective tissue (7).

In the present study, the entire coding region of $F B N 1$ was analyzed, and a novel mutation in exon 14 of $F B N 1$ was identified in all affected members. The newly identified FBN1 mutation in a Chinese family with MFS further emphasizes the important role of FBN1 in the mechanism of MFS development. The present study not only expanded the 
mutation spectrum of $F B N 1$ resulting in MFS development in a Chinese family, but is also likely to aid understanding of the molecular pathogenesis and clinical diagnosis of FBN1-associated MFS.

\section{Materials and methods}

Subjects. A family with MFS was recruited from the Shandong Provincial Hospital Affiliated to Shandong University (Jinan, China) (Fig. 1). This study was conducted in accordance to the tenets of The Declaration of Helsinki and was approved by the Institutional Review Boards of the Hospital of University of Electronic Science and Technology of China and Sichuan Provincial People's Hospital (Chengdu, China), and the Shandong Provincial Hospital Affiliated to Shandong University. A total of 383 ethnically matched, unrelated and normal healthy individuals were recruited from the Hospital of University of Electronic Science and Technology of China \& Sichuan Provincial People's Hospital (255 males and 128 females; mean age at recruitment $55.26 \pm 8.78$ years). These control individuals had no medical history associated with any related diseases. Written informed consent was obtained from all participants prior to the study.

Clinical diagnosis. Two of the family members were diagnosed with MFS according to the revised Ghent criteria (2). Non-consanguineous marriages were found in the family; clinical information of the affected family members is summarized in Table I. All members of this family underwent complete physical, cardiovascular and ophthalmologic examinations. Unrelated healthy individuals also underwent the same examinations.

Mutation screening. Genomic DNA samples were extracted from peripheral blood using a Blood DNA extraction kit (Thermo Fisher Scientific, Inc., Waltham, MA, USA). The whole coding region of FBN1 (NM_000138.4) was amplified by polymerase chain reaction (PCR) with 35 cycles $(30 \mathrm{sec}$ at $95^{\circ} \mathrm{C}$ for initial denaturation, $30 \mathrm{sec}$ for annealing at different temperatures as shown in Table II, and $30 \mathrm{sec}$ at $72^{\circ} \mathrm{C}$ for extension), using a GeneAmp ${ }^{\circledR}$ PCR system 9700 (Applied Biosystems; Thermo Scientific Inc.). Sequencing primers of all the exons were designed using Primer 5.0 (Premier Biosoft International, Palo Alto, CA, USA; Table II). Amplified PCR products were purified and sequenced directly (BigDye Terminators Sequencing kit) with an Automated Genetic Analysis system 3130 (both from Applied Biosystems; Thermo Fisher Scientific, Inc.). Comparative amino acid sequence analysis of the human FBN1 protein was performed across different species using HomoloGene (https://www.ncbi.nlm.nih .gov/homologene/?term=FBN1). The potentially damaging effects of the mutation on the structure and function of FBN1 was predicted using SIFT (http://sift.jcvi.org) and PolyPhen-2 (http://genetics.bwh.harvard.edu/pph2/).

\section{Results}

Clinical findings. The parents and two daughters of a family from Shandong, China, were included in the present study (Fig. 1). Other relatives of this family were not willing
Table I. Clinical details of the patients with Marfan syndrome in the family.

\begin{tabular}{|c|c|c|}
\hline Characteristic & Proband (I:1) & $\begin{array}{c}\text { Proband's } \\
\text { daughter (II:2) }\end{array}$ \\
\hline Age (years) & 44 & 8 \\
\hline Gender & M & $\mathrm{F}$ \\
\hline Ectopialentis & + & + \\
\hline Myopia & + & + \\
\hline Strabismus & + , exotropia & + , exotropia \\
\hline Glaucoma & - & - \\
\hline Retinal detachment & + & - \\
\hline Height $(\mathrm{cm})$ & 184 & 134 \\
\hline Arm span (cm) & 186 & 137 \\
\hline $\mathrm{AS} / \mathrm{H}$ & 1.01 & 1.02 \\
\hline $\begin{array}{l}\text { Overgrowth of } \\
\text { the long bones }\end{array}$ & + & + \\
\hline Arachnodactyly & + & + \\
\hline Scoliosis & - & - \\
\hline Pectus excavatum & - & - \\
\hline Pectus carinatum & + & - \\
\hline Flatfeet & + & + \\
\hline Mitral valve prolapse & - & - \\
\hline Aortic aneurysm & $\begin{array}{l}+ \text { (ruptured } 5 \text { years } \\
\text { ago then formed } \\
\text { aortic dissection; } \\
\text { Bentall surgery } \\
\text { was performed at } \\
\text { that time) }\end{array}$ & - \\
\hline $\begin{array}{l}\text { Aortic root } \\
\text { dimension }(\mathrm{mm})\end{array}$ & $\begin{array}{c}25.0 \text { (artificial } \\
\text { vessel diameter) }\end{array}$ & 29.1 \\
\hline
\end{tabular}

M, male; F, female; AS, arm span; H, Height.

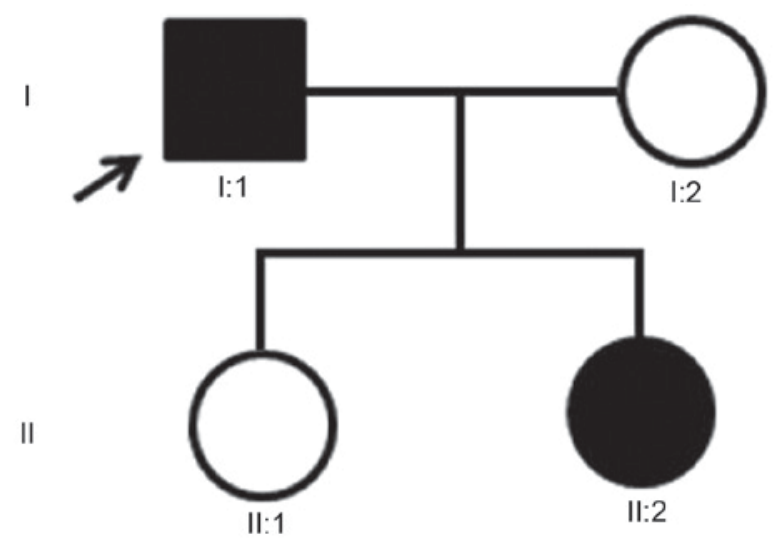

Figure 1. Pedigree of the family with Marfan syndrome. Solid symbols indicate affected patients, open symbols indicate unaffected subjects and arrow indicates the proband in this family. Squares represent males and circles represent females.

to be tested and so additional clinical details were unattainable. Two affected patients (I:1 and II:2) exhibited similar 
Table II. Primers used for mutation screening of the FBN1 gene.

\begin{tabular}{|c|c|c|c|}
\hline Primer name & Primer sequence $\left(5^{\prime}-3^{\prime}\right)$ & Product size (bp) & Annealing temperature $\left({ }^{\circ} \mathrm{C}\right)$ \\
\hline$F B N 11 \& 2 F$ & TCGGGGATTTGTCTCTGTGT & 434 & 59 \\
\hline$F B N 11 \& 2 R$ & GCCCGTTGTTCTGGATCTTG & & \\
\hline$F B N 13 F$ & ACCAACCCAGCATTGAGTCT & 308 & 60 \\
\hline FBN13R & TTCTAAGGCTCCCCATGCAA & & \\
\hline$F B N 14 F$ & TTGTGAGGGACCTGAGAACC & 296 & 59 \\
\hline FBN14R & TTGCAGGAAAGAGGAAAGCC & & \\
\hline$F B N 15 F$ & CAACTCCTGTGAGCTGTTGC & 278 & 60 \\
\hline FBN15R & AAACATGCTGTGTCCCAGGT & & \\
\hline$F B N 16 F$ & GTCCTTCCAGAGGACCACAA & 228 & 60 \\
\hline$F B N 16 R$ & CAGCTTTAGGTACCAGCATGTC & & \\
\hline$F B N 17 F$ & GCATGATGGTTCCTGCTTTT & 380 & 60 \\
\hline FBN17R & GCAGTCAGCGAAATTGTGAA & & \\
\hline$F B N 18 F$ & TTCCAAATATTGTGATGGACAAA & 448 & 60 \\
\hline FBN18R & ACAGGGTTTTTCTGGTCCAA & & \\
\hline$F B N 19 F$ & GCTGTTTCCAGGGACATGAT & 441 & 60 \\
\hline FBN19R & TTTATGGGAGGCAAAACGTC & & \\
\hline FBN110F & AGCCCCAGTGTGAAGTATGG & 396 & 60 \\
\hline FBN110R & TTCCCTGGACGTCATCTCTT & & \\
\hline$F B N 111 F$ & TGACTTCTGTGGGCCTATGA & 300 & 59 \\
\hline FBN111R & TTAACTTGAACAATGCAAGAAAAA & & \\
\hline$F B N 112 F$ & TTGTCACCAGACGACCTTTG & 383 & 60 \\
\hline FBN112R & CCACCAAGTTTGGGGTAAGTT & & \\
\hline FBN113F & AAAAGGAACCCAGAAAGTCTTAGAA & 295 & 60 \\
\hline$F B N 113 R$ & CTTCCGGCATGGGTTATTTA & & \\
\hline FBN114F & GGAGGGAGGGGGAAATAAA & 244 & 60 \\
\hline FBN114R & ACTGCAATGGAAGGAGAGGA & & \\
\hline FBN115F & GATCTTATTTGGATGAAAGTTAGCC & 400 & 59 \\
\hline FBN115R & AGTCAGGTTTCCCAAACCAA & & \\
\hline$F B N 116 F$ & TTCCCCATTTTCAAGGGTTA & 294 & 61 \\
\hline FBN116R & CGTTTGTTACCATTGGGCTTT & & \\
\hline$F B N 117 F$ & GGGGGTTCTCATCTGTTTGA & 242 & 60 \\
\hline FBN117R & CAGTACGAGGGCATCTCCAT & & \\
\hline$F B N 118 F$ & ACCAAGGGCAGGATCTACCT & 188 & 60 \\
\hline FBN118R & ACCCACAAGAAAGCCTGATG & & \\
\hline FBN119F & CCTGTAGCTCCTAAGGTCATTACA & 300 & 60 \\
\hline FBN119R & CTCCCAGCAATGAAAGAAGG & & \\
\hline FBN120F & CAAAGTTTGGGCCCTTTTTA & 226 & 59 \\
\hline FBN120R & TGGCATTCCAAAAGATAGCA & & \\
\hline FBN121F & GGCCCAAGACTAGATTTTAGCA & 243 & 60 \\
\hline FBN121R & TTTTGCAGGAAAAGCTGACA & & \\
\hline FBN122F & AATGTCAGCTTTTCCTGCAA & 368 & 59 \\
\hline FBN122R & TGAAATACTAGGCTTCCCCTTT & & \\
\hline$F B N 123 F$ & TGTCAGAACTGCAAAGTCTGG & 204 & 60 \\
\hline FBN123R & GACAGCTTTATCCAGTCCGAGT & & \\
\hline FBN124F & TGCTATTCAGGCACCCTAGA & 400 & 59 \\
\hline FBN124R & TGGAGTGTGTGTCTGTACCTGA & & \\
\hline FBN125F & AACAGAGTGTTGGCAGTTTGG & 373 & 60 \\
\hline FBN125R & CTGAGATCATGAAAATGCATCC & & \\
\hline$F B N 126 \& 27 F$ & GACCTCCTGACTGCTTGCTC & 494 & 60 \\
\hline$F B N 126 \& 27 R$ & CAAAGCTTCATGGAATCCTTCT & & \\
\hline$F B N 128 \& 29 F$ & GAGTGCTTGGTCTGGTGGAG & 564 & 61 \\
\hline$F B N 128 \& 29 R$ & AGCGATGAAAACAAAACTCAGA & & \\
\hline
\end{tabular}


Table II. Continued.

\begin{tabular}{|c|c|c|c|}
\hline Primer name & Primer sequence (5'-3') & Product size (bp) & Annealing temperature $\left({ }^{\circ} \mathrm{C}\right)$ \\
\hline FBN130F & GGGACAGACATCCAAACCAT & 249 & 62 \\
\hline FBN130R & CAAAGCCTGGGCCCTAAAC & & \\
\hline FBN131F & CTCACTGAACAGTGGAACCAA & 280 & 59 \\
\hline FBN131R & GCTCTCTTTGGAATGCTGGT & 280 & 59 \\
\hline$F B N 132 F$ & GAATCTTTCTATCACTGACCCAAAC & & \\
\hline FBN132R & TCGAGGGGAAAGTACTCAATG & 325 & 59 \\
\hline$F B N 133 \& 34 F$ & CATTTGTGCTGAGCCTTTTTC & 495 & 60 \\
\hline$F B N 133 \& 34 R$ & GAATGCCTGGCTTCTCTGAC & & \\
\hline FBN135F & TGCTGCACTGGAAAGTTGAT & 231 & 60 \\
\hline FBN135R & AGTGGCTTCCCCATCAGTTA & & \\
\hline$F B N 136 F$ & TGCCCAGATTGGTGTTAGAT & 400 & 59 \\
\hline FBN136R & CAGGTCTGAGAAAAGGTATCTGTG & & \\
\hline FBN137\&38F & AGATTGGGCCCTGTTCTTTT & 819 & 60 \\
\hline$F B N 137 \& 38 R$ & TTGGGAATAAGGTCCCCTCT & & \\
\hline FBN139\&40F & TCAGACGGGCAGAGTAACAA & 496 & 59 \\
\hline FBN139\&40R & CCATATTCTGGTTTTGCAGGT & & \\
\hline FBN141F & AGGCCATTCCAAAATGTGAA & 249 & 60 \\
\hline FBN141R & TTGTGAGCTCTCTTCCTCTTTGT & & \\
\hline$F B N 142 F$ & ATTTCCCACATGGCATCAC & 300 & 60 \\
\hline FBN142R & TGCTTCCTTCGCTAAGACTGA & & \\
\hline FBN143F & СТАТССТСССАТСССАССТТ & 273 & 60 \\
\hline FBN143R & CAGGGTGTTTGCACAGTTTG & & \\
\hline FBN144F & CACAGGGATCATGTGCTGTC & 315 & 60 \\
\hline FBN144R & TCCACACCATGCCCTTTACT & & \\
\hline FBN145F & GGCTTTGTTGACTGGACACC & 218 & 62 \\
\hline FBN145R & GTAGGCATGTCCAGCCTGTG & & \\
\hline FBN146F & GAGCTAGGATTACTCCTGAGAATGA & 398 & 59 \\
\hline FBN146R & TCATGTTCAGATTGCCAAAGA & & \\
\hline$F B N 147 F$ & GGCCTGGTGAACCCTAAAAT & 247 & 60 \\
\hline FBN147R & TTCCTTTGCTGATGCACAAT & & \\
\hline FBN148F & TGCTGGGATTATGACATCTTTG & 292 & 60 \\
\hline FBN148R & TTTTCCTCCAGGTTTCCAGA & & \\
\hline FBN149F & CCAGTGGGAACCTCTTCCTT & 205 & 60 \\
\hline FBN149R & GACACCCGACACTCCTCATT & & \\
\hline FBN150F & TGATGTCTCCATCGTGTTTTG & 208 & 61 \\
\hline FBN150R & ATTGAAAGCCCAAAGCCTTC & & \\
\hline FBN151F & GGAAAGCAACTGAAGGGTGT & 263 & 590 \\
\hline FBN151R & GCCTACAGTCTTACTTACATCATGG & & \\
\hline$F B N 152 \& 53 F$ & GGAGAAGCTTGTAATGAATTGCT & 594 & 60 \\
\hline$F B N 152 \& 53 R$ & AACTTATTTCAGTGCCATCTTGG & & \\
\hline FBN154F & TTTGGACACATTCCTGGTTTC & 207 & 60 \\
\hline FBN154R & CAACCAATTGTTCCCAGGAT & & \\
\hline FBN155F & CCTTTTGTTGCTGTCCATGAT & 249 & 60 \\
\hline FBN155R & AGGGAAGCTTTGAGGGACAT & & \\
\hline FBN156F & TCATACTCAACAGAGCAGAAGGA & 363 & 59 \\
\hline FBN156R & CAAGAACTCAGAGCCCAGGT & & \\
\hline FBN157F & AAGGAACAAAGGGAGGGAAG & 392 & 60 \\
\hline FBN157R & CAGTCATTACGGCATCTCCA & & \\
\hline FBN158F & CTGACATCCCCTTTGCCATA & 277 & 61 \\
\hline FBN158R & TCCCTGCAAGTATTTTTGGAC & & \\
\hline FBN159\&60F & CACTGAAGTGACCCCCTACA & 600 & 60 \\
\hline$F B N 159 \& 60 R$ & TGAGGGGCAATGGTCAAT & & \\
\hline
\end{tabular}


Table II. Continued.

\begin{tabular}{|c|c|c|c|}
\hline Primer name & Primer sequence $\left(5^{\prime}-3^{\prime}\right)$ & Product size (bp) & Annealing temperature $\left({ }^{\circ} \mathrm{C}\right)$ \\
\hline FBN161\&62F & TGTTGGCTTGACTCAAATGC & 600 & 61 \\
\hline FBN161\&62R & CСTCCACAAGGATTCACCAG & & \\
\hline FBN163F & TGGTGGCTCTGCTTCTTTTT & 178 & 60 \\
\hline FBN163R & GCCATGCATCTTGAGAGTGA & & \\
\hline FBN164F & AAGTGGCCAGATCCAATGTC & 334 & 60 \\
\hline FBN164R & ACCATGACCAGGAAGAGCAC & & \\
\hline FBN165F & CATCTATGCTCCССТTCTGC & 243 & 60 \\
\hline FBN165R & TTCCACCACAGGAGACATCA & & \\
\hline FBN166F & GCAGCATAAGGCAGAAAATTG & 583 & 60 \\
\hline FBN166R & TGATTCTGATTGGGGGAAAA & & \\
\hline
\end{tabular}

FBN1, fibrillin-1; F, forward; R, reverse; bp, base pair.
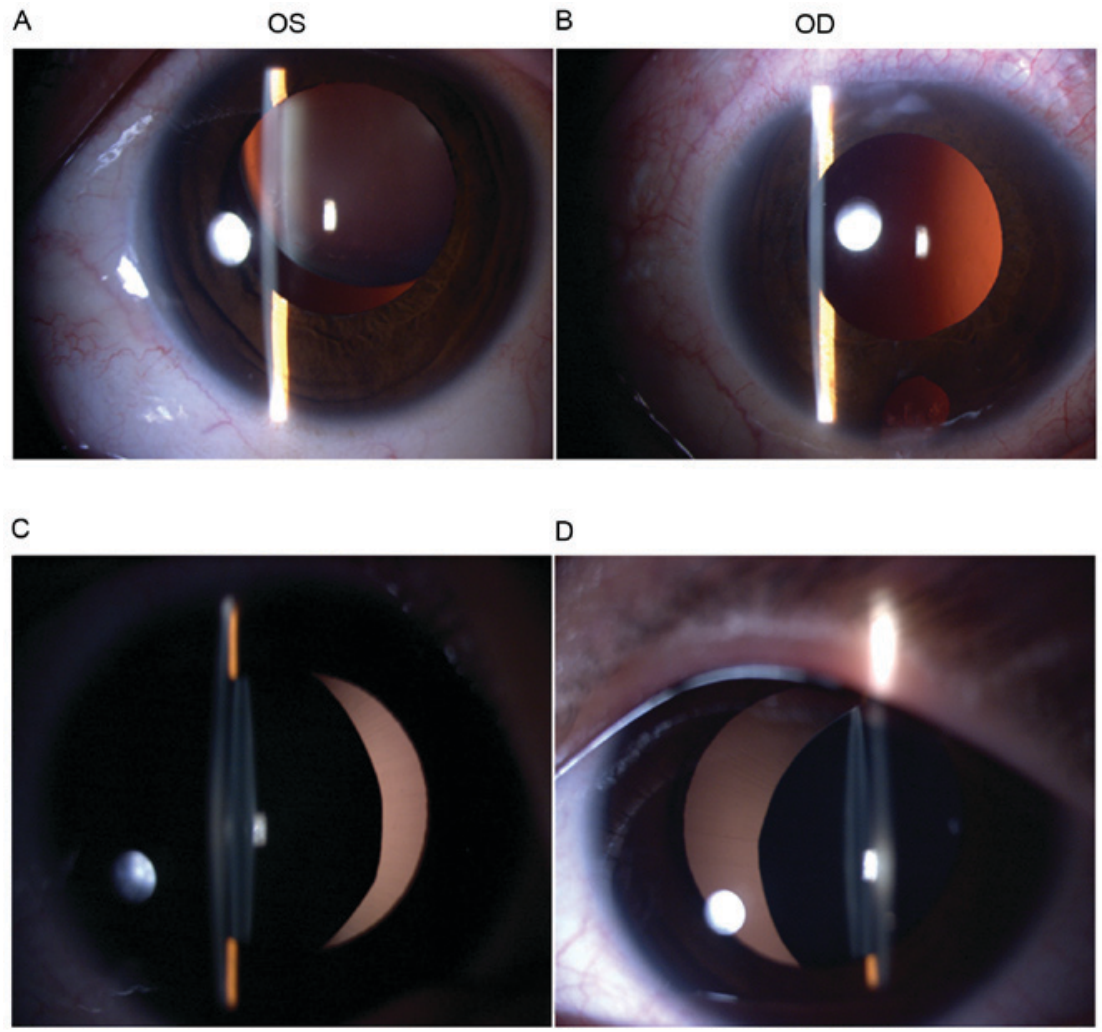

Figure 2. Slitlamp photograph of the proband (I:1) and his daughter (II:2) in the family with MFS. (A) Left eye of the proband had ectopialentis. (B) Lensectomy and vitrectomy combined with silicone oil tamponade was performed for the right eye of the proband following retinal reattachment and silicone oil removed 3 months later. (C and D) Ectopialentis of the proband's daughter (II:2). (C) Lens nasal deviation occurred in her left eye and (D) nasal-inferior dislocation in her right eye. OS, oculus sinister (left eye); OD, oculus dexter (right eye).

clinical symptoms, including ectopialentis, myopia and strabismus (Fig. 2 and Table I). The left eye of the proband (I:1) underwent refractive lensectomy and vitrectomy combined with silicone oil tamponade after retinal detachment 2 years prior to the current study; following retinal re-attachment, silicone oil was removed 3 months later. The two patients both had the same facial and skeletal features, including arachnodactyly, flat feet and dilation of the aortic root (Fig. 3 and Table I). The proband had pectus carinatum and aortic aneurysm. The patient received Bentall surgery and underwent aortic arch replacement 5 years prior to the current study, as their aortic aneurysm ruptured and formed aortic dissection (Fig. 4). The other two members of the family had no features of MFS.

Mutation screening of FBN1. Direct sequencing of the whole coding region of $F B N 1$ detected a novel missense mutation c. $1708 \mathrm{~T}>\mathrm{G}$ (p.C570G), situated at nucleotide 570 in exon 14 of the coding region (Fig. 5A). This heterozygous mutation was detected in the two affected patients (I:1 and II:2) but was not 
A

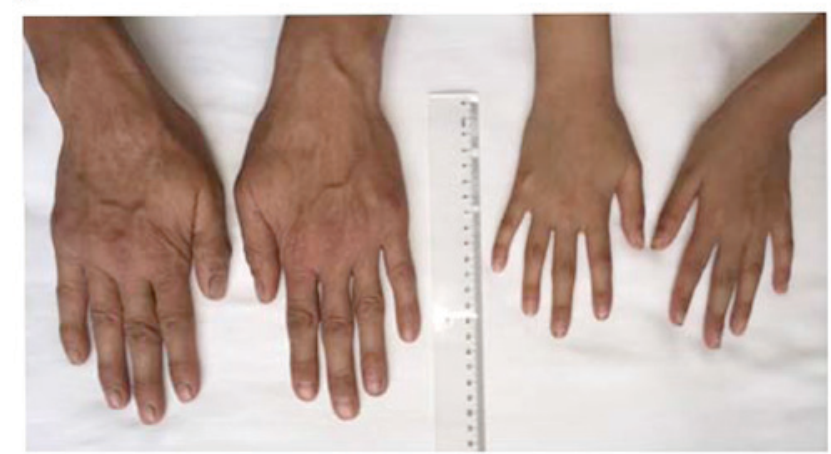

B

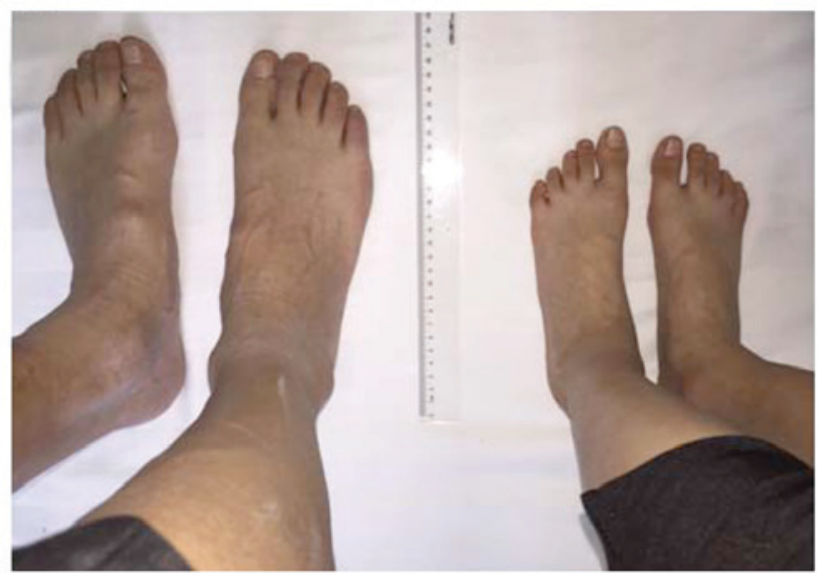

Figure 3. Arachnodactyly of the proband (I:1) and the affected daughter (II:2). (A) Proband (left) and his daughter (right) had (B) long fingers and (B) flat feet.

found in the unaffected mother and daughter (I:2 and II:1) of the family and in the 383 ethnically matched healthy subjects. Therefore, c. $1708 \mathrm{~T}>\mathrm{G}$ (p.C570G) cosegregated to the patients with MFS in this family. Multiple sequencing alignment of human FBN1 protein with various species revealed that the novel mutation occurred within a highly conserved region of the calcium binding epidermal growth factor-like (cbEGF) domain (Fig. 5C). This mutation is a $\mathrm{T}>\mathrm{G}$ transition, converting cysteine to glycine at amino acid 570 (p.C570G). This amino acid substitution in the FBN1 protein was predicted to be damaging by SIFT and PolyPhen-2.

\section{Discussion}

It has been reported that MFS is mainly caused by mutations in the FBN1 gene, which was the first gene identified to cause MFS (8). Of all the identified mutations in the FBN1 gene, $38.6 \%$ result in a truncated FBN1 protein and $60.3 \%$ represent missense mutations across different ethnic groups (9). FBN1 mutations may cause abnormalities in the formation of microfibrils and fibrillin. As a result, connective tissues weaken (10). A novel FBN1 heterozygous missense mutation, c.1708 T>G (p.C570G) was identified within a Chinese family associated with MFS in the present study.

FBN1 is an important component of microfibrils and is expressed in many human tissues, including in zonules, the cardiovascular system, cartilage, tendon and cornea. The
A

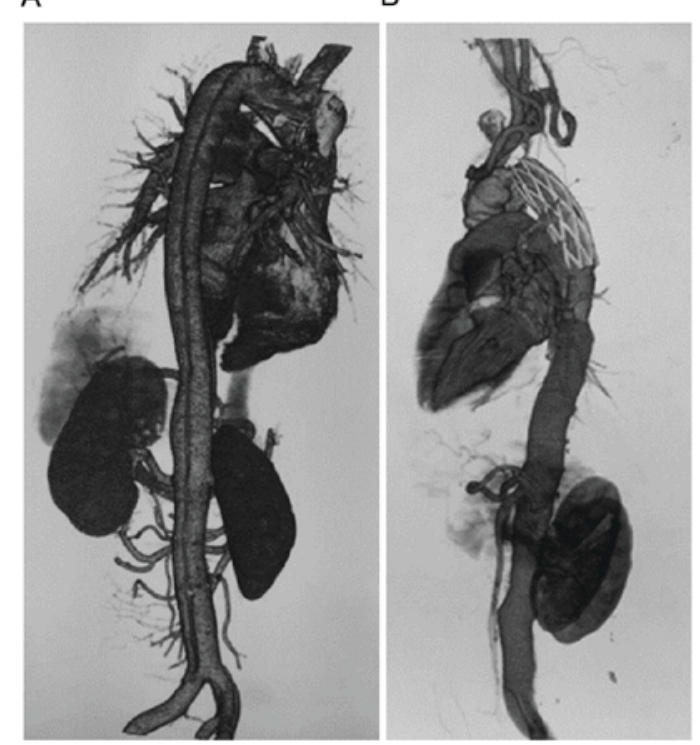

Figure 4. CTA of the aortic vessels of the proband. (A) CTA confirmed the formation of aortic dissection. (B) CTA image of the artificial vessel of the proband after Bentall surgery. CTA, computed tomography angiogram.

protein serves a role in the formation of zonules and is secreted from ciliary bodies of non-pigmented cells (11). FBN1 protein is composed of repeated modules, including cbEGF and transforming growth factor-1 binding protein-like domains, and is responsible for maintaining microfibers in an ordered arrangement $(12,13)$. The majority of identified missense mutations in $F B N 1$ are localized in cbEGF (14). The mutated monomer of FBN1 could interfere with the polymerization of fibrillin and microfiber aggregation (15). FBN1 mutations within cbEGF modules may disrupt the stability of elastic fibers and render FBN1 susceptible to proteolysis. As a result, the transforming growth factor- $\beta$ signaling activity that affects extracellular matrix formation may malfunction $(4,16)$.

In the present study, a novel c.1708 T>G (p.C570G) heterozygous missense mutation of the $F B N 1$ gene was reported in a Chinese family with MFS. Three similar missense mutations: c.1709G $>$ A (p.C570Y) (17), c.1709G $>$ C (p.C570S) (18) and c.1709G $>C$ (p.C570R) (19) have been reported in sporadic cases; however, clinical data in these studies were not obtained. In this pedigree, c. $1708 \mathrm{~T}>\mathrm{G}$ (p.C570G) in FBN1 was detected in the two patients with MFS (I:1 and II:2).The proband (I:1) initially came to Shandong Provincial Hospital to see an ophthalmologist and was found to suffer from ectopialentis, myopia and strabismus in both eyes. The proband and the affected daughter (II:2) had similar facial and skeletal features of MFS, including arachnodactyly, flat feet and dilation of aortic root. In addition, pectus carinatum, aortic dissection and retinal detachment were also detected in the proband. These findings suggested that the clinical manifestations of the patient with MFS became more evident with age. This mutation was not included in the Exome Aggregation Consortium dataset; c.1708 T>G (p.C570G) of FBN1 was not detected in the mother (I:2) and another daughter (II:1) of this family, or in the 383 unrelated normal controls during the mutation screening in the present study. This indicated that c.1708 T>G (p.C570G) of FBNl cosegregated with affected MFS patients 
A

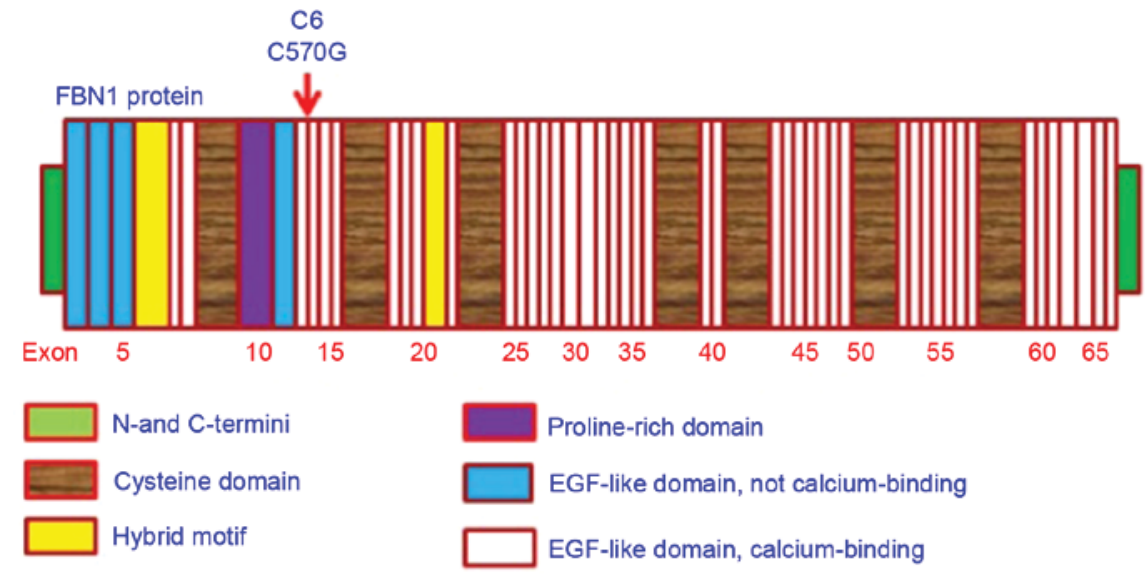

B

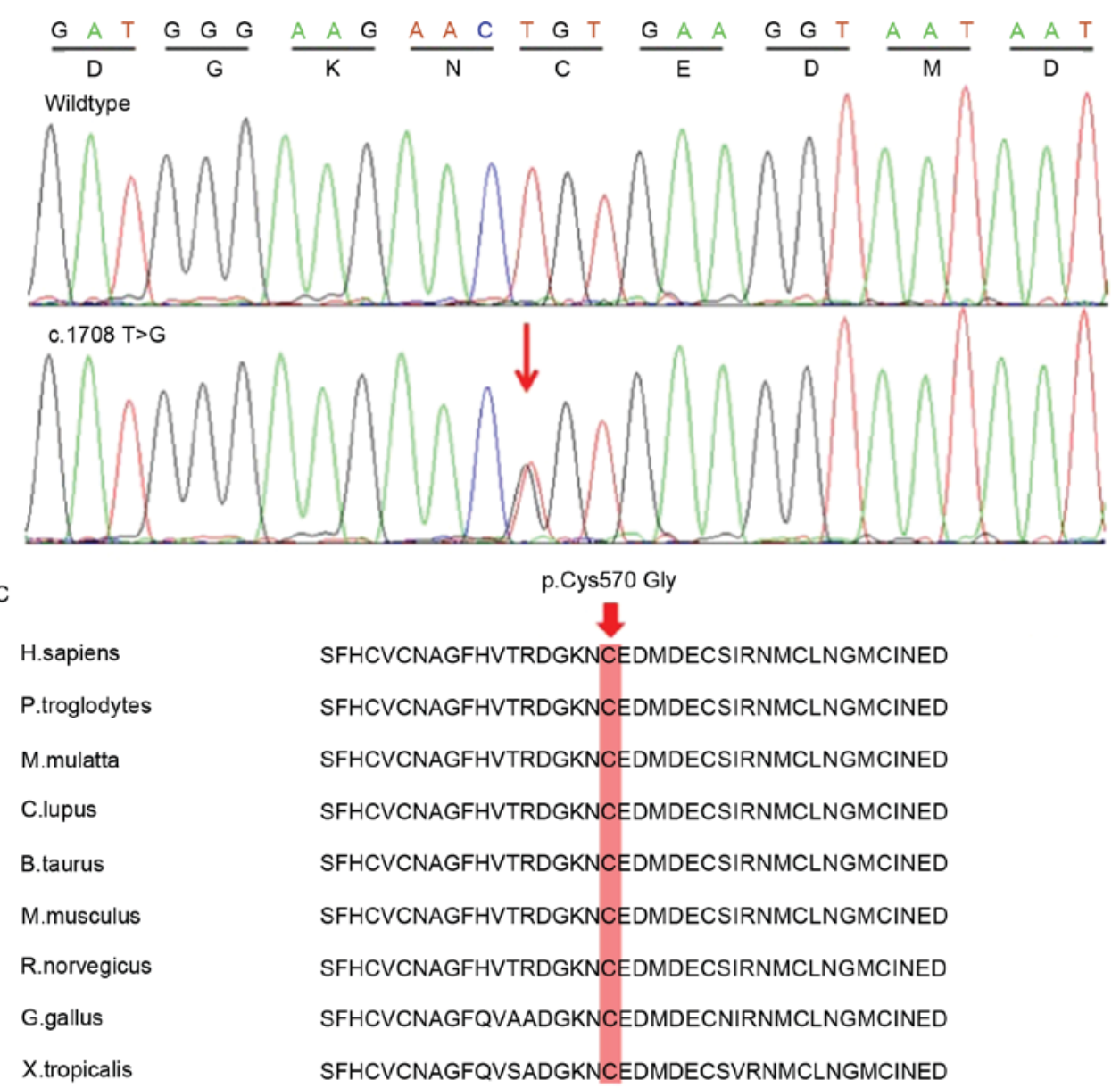

Figure 5. Representative chromatogram of FBN1 sequence. (A) FBN1 has different functional regions and the p.C570G mutation occurs in the calcium binding EGF-like domain. (B) Normal sequence from an unaffected individual (I:2) (upper sequence), and a heterozygous $\mathrm{T}$ to $\mathrm{G}$ substitution at codon 570 from affected subjects (I:1 and II:2) (lower sequence). (C) Orthologous protein sequence alignment of FBN1 from different species, the mutated residue showing conservation of cysteine at codon 570 was shaded in red. EGF, epidermal growth factor; FBN1, fibrillin-1.

and may serve an important role in the pathogenesis of MFS development in this pedigree.

The p.C570G mutation of FBN1 identified in this family with MFS resulted in a substitution of a highly conserved cysteine residue for glycine in a cbEGF domain of FBN1. This mutation is predicted to abolish one disulfide bond and thus affect the sixth conserved cysteine (C6) of the cbEGF domain; disulfide bonds are essential for the correct EGF-like domain structure. SIFT and PolyPhen-2 predictions indicated that this mutation is critical to protein function, supporting a possible pathogenic effect of this mutation. Evidence has revealed that most $F B N 1$ mutations are clustered in exons 24-32, a hot spot region associated with classic and severe forms of MFS $(17,20)$; mutations in exons 12-15 encoding cbEGF-like domains (C3-C6) cause a mild phenotype of MFS with possible late cardiovascular involvement (21). Evidence from the present study consistently indicated that the identified heterozygous mutation, c. $1708 \mathrm{~T}>\mathrm{G}$, is located at exon 14 and 
that this cysteine substitution detected in the proband resulted in pectus carinatum and aortic dissection. These two factors correlated with increasing age. However, evident symptoms were not detected in the young affected daughter (II:2), even though significant dilation of the aortic root was identified. Nevertheless, further functional analyses are required to confirm the role of FBN1 and its underlying mechanisms in MFS.

In conclusion, a novel heterozygous mutation, c.1708 $\mathrm{T}>\mathrm{G}$ (p.C570G), in the FBN1 gene was identified in a Chinese family associated with MFS. The results from the present study enrich the spectrum of MFS-associated mutations of FBN1 and may aid presymptomatic molecular diagnosis of undetermined cases of MFS.

\section{Acknowledgements}

The present study was supported by grants from the Natural Science Foundation of China [grant nos. 81670853 (B.G) and 81371048 (B.G)], the Department of Science and Technology of Sichuan Province [grant no. 2015HH0031 (B.G.)] and the Health and Family Planning Commission of Sichuan Province of China [grant no. 16ZD028 (B.G.)].

\section{References}

1. Judge DP and Dietz HC: Marfan's syndrome. Lancet 366: 1965-1976, 2005.

2. Radonic T, de Witte P, Groenink M, de Bruin-Bon RA, Timmermans J, Scholte AJ, van den Berg MP, Baars MJ, van Tintelen JP, Kempers M, et al: Critical appraisal of the revised Ghent criteria for diagnosis of Marfan syndrome. Clin Genet 80: 346-353, 2011.

3. Zhao F, Pan X, Zhao K and Zhao C: Novel mutations of fibrillin-1 gene correlate with different phenotypes of Marfan syndrome in Chinese families. Mol Vis 19: 751-758, 2013.

4. Boileau C, Jondeau G, Mizuguchi T and Matsumoto N: Molecular genetics of Marfan syndrome. Curr Opin Cardiol 20: 194-200, 2005.

5. Baetens M, Van Laer L, De Leeneer K, Hellemans J, De Schrijver J, Van De Voorde H, Renard M, Dietz H, Lacro RV, Menten B, et al: Applying massive parallel sequencing to molecular diagnosis of Marfan and Loeys-Dietz syndromes. Hum Mutat 32: 1053-1062, 2011.

6. Faivre L, Collod-Beroud G, Child A, Callewaert B, Loeys BL, Binquet C, Gautier E, Arbustini E, Mayer K, Arslan-Kirchner M, et al: Contribution of molecular analyses in diagnosing Marfan syndrome and type I fibrillinopathies: An international study of 1009 probands. J Med Genet 45: 384-390, 2008.

7. Vollbrandt T, Tiedemann K, El-Hallous E, Lin G, Brinckmann J, John H, Bätge B, Notbohm H and Reinhardt DP: Consequences of cysteine mutations in calcium-binding epidermal growth factor modules of fibrillin-1. J Biol Chem 279: 32924-32931, 2004.
8. Dietz HC,Cutting GR, Pyeritz RE, Maslen CL, Sakai LY, Corson GM, Puffenberger EG, Hamosh A, Nanthakumar EJ, Curristin SM, et al: Marfan syndrome caused by a recurrent de novo missense mutation in the fibrillin gene. Nature 352: 337-339, 1991.

9. Faivre L, Collod-Beroud G, Loeys BL, Child A, Binquet C, Gautier E, Callewaert B, Arbustini E, Mayer K, Arslan-Kirchner M, et al: Effect of mutation type and location on clinical outcome in 1,013 probands with Marfan syndrome or related phenotypes and FBN1 mutations: An international study. Am J Hum Genet 81: 454-466, 2007.

10. Cañadas V, Vilacosta I, Bruna I and Fuster V: Marfan syndrome. Part 1: Pathophysiology and diagnosis. Nat Rev Cardiol 7: 256-265, 2010.

11. Dureau P: Pathophysiology of zonular diseases. Curr Opin Ophthalmol 19: 27-30, 2008.

12. Whiteman P and Handford PA: Defective secretion of recombinant fragments of fibrillin-1: Implications of protein misfolding for the pathogenesis of Marfan syndrome and related disorders. Hum Mol Genet 12: 727-737, 2003.

13. Werner JM, Knott V, Handford PA, Campbell ID and Downing AK: Backbone dynamics of a cbEGF domain pair in the presence of calcium. J Mol Biol 296: 1065-1078, 2000.

14. Dietz HC, Saraiva JM, Pyeritz RE, Cutting GR and Francomano CA: Clustering of fibrillin (FBN1) missense mutations in Marfan syndrome patients at cysteine residues in EGF-like domains. Hum Mutat 1: 366-374, 1992.

15. Dietz HC, McIntosh I, Sakai LY, Corson GM, Chalberg SC, Pyeritz RE and Francomano CA: Four novel FBN1 mutations: Significance for mutant transcript level and EGF-like domain calcium binding in the pathogenesis of Marfan syndrome. Genomics 17: 468-475, 1993.

16. Mizuguchi T, Collod-Beroud G, Akiyama T, Abifadel M, Harada N, Morisaki T, Allard D, Varret M, Claustres M, Morisaki H, et al: Heterozygous TGFBR2 mutations in Marfan syndrome. Nat Genet 36: 855-860, 2004.

17. Loeys B, Nuytinck L, Delvaux I, De Bie S and De Paepe A: Genotype and phenotype analysis of 171 patients referred for molecular study of the fibrillin-1 gene FBN1 because of suspected Marfan syndrome. Arch Intern Med 161: 2447-2454, 2001.

18. Ogawa N, Imai Y, Takahashi Y, Nawata K, Hara K, Nishimura H, Kato M, Takeda N, Kohro T, Morita H, et al: Evaluating Japanese patients with the Marfan syndrome using high-throughput microarray-based mutational analysis of fibrillin-1 gene. Am J Cardiol 108: 1801-1807, 2011.

19. Schrijver I, Liu W, Brenn T, Furthmayr H and Francke U: Cysteine substitutions in epidermal growth factor-like domains of fibrillin-1: Distinct effects on biochemical and clinical phenotypes. Am J Hum Genet 65: 1007-1020, 1999.

20. Tiecke F, Katzke S, Booms P, Robinson PN, Neumann L, Godfrey M, Mathews KR, Scheuner M, Hinkel GK, Brenner RE, et al: Classic, atypically severe and neonatal Marfan syndrome: Twelve mutations and genotype-phenotype correlations in FBN1 exons 24-40. Eur J Hum Genet 9: 13-21, 2001.

21. Pepe G, Lapini I, Evangelisti L, Attanasio M, Giusti B, Lucarini L, Fattori R, Pellicanò G, Scrivanti M, Porciani MC, et al: Is ectopia lentis in some cases a mild phenotypic expression of Marfan syndrome? Need for a long-term follow-up. Mol Vis 13: 2242-2247, 2007. 